\title{
Epidemiology incidence and geographical distribution of Pertussis using GIS and its incidence prediction in Iran in 2021
}

\author{
Seyed Mohsen Zahraei ${ }^{1}$, Parvin Mohamadi ${ }^{2}$, Ghobad Moradi ${ }^{3}$, Samira Shirzadi ${ }^{4}$, Fatemeh Azimian ${ }^{5}$, Zaher Khazaei ${ }^{6}$, \\ Hasan Naemi ${ }^{7}$, Elham Goodarzi ${ }^{8 *} \mathbb{D}$
}

Received: 14 Feb 2020

Published: 24 Aug 2021

\section{Abstract}

Background: Pertussis is a respiratory tract infection caused by Bordetella pertussis, which causes inflammation of the lungs and respiratory tract. The purpose of this study was to investigate the incidence and geographical distribution of pertussis using the geographic information system (GIS) and to predict its incidence in Iran in 2021.

Methods: This was a descriptive analytical study. Information on pertussis was obtained from the Center for Communicable Diseases Control during 2009-2015. In the next step, the ArcGIS 9.3 was used to prepare geographic maps of the disease incidence and frequency. Therefore, the disease prediction map was drawn. using the Raster Calculator tool.

Results: The results showed that the highest incidence of pertussis during 2009-2015 was in Zanjan, Qom, Mazandaran, and Qazvin provinces. The incidence of pertussis in Iran increased from 0.74 in 2009 to 1.53 in 2015. Based on the modeling results for Iran, Qom, Mazandaran, Tehran, Qazvin, and Zanjan provinces, with 76.76\%, 73.69\%, 66.32\%, 30.94\% and 24.18\% of their areas (Km2), are at high risk for pertussis in the coming years, respectively.

Conclusion: The incidence of the disease has been increasing in recent years, indicating the emergence of the disease in Iran. The modeling maps show that the Iranian provinces of Qom, Tehran, Zanjan, and Qazvin are at risk of the disease incidence in the coming years, indicating the need for planning, appropriate interventions and more precise implementation of the vaccination program against the disease.

Keywords: Incidence, Prediction, Pertussis, GIS, Iran

Conflicts of Interest: None declared

Funding: Kurdistan University of Medical Sciences

\section{*This work has been published under CC BY-NC-SA 1.0 license. \\ Copyright $\odot$ Iran University of Medical Sciences}

Cite this article as: Zahraei SM, Mohamadi P, Moradi G, Shirzadi S, Azimian F, Khazaei Z, Naemi H, Goodarzi E. Epidemiology incidence and geographical distribution of Pertussis using GIS and its incidence prediction in Iran in 2021. Med J Islam Repub Iran. 2021 (xx);35:x. https://doi.org/10.47176/mjiri.35.x

\section{Introduction}

Pertussis is a highly contagious acute respiratory disease caused by the pathogen of Bordetella pertussis. The dis-

Corresponding author: Elham Goodarzi, goodarzi.e@lums.ac.ir

1. Center for Communicable Diseases Control, Ministry of Health and Medical Education, Tehran, Iran

2. Department of Medical Sciences, Sanandaj Branch, Islamic Azad University, Sanandaj, Iran

3. Social Determinants of Health Research Center, Research Institute for Health Development, Kurdistan University of Medical Sciences, Sanandaj, Iran

4. Payame Noor University, Tehran, Iran

5. Iranian Center for Communicable Disease Control, Ministry of Health \& Medical Education, Tehran, Iran

6. Department of Public Health, School of Medicine, Dezful University of Medical Sciences, Dezful, Iran

7. Cellular and Molecular Research Center, Sabzevar University of Medical Sciences, Sabzevar, Iran

${ }^{8 .}$ Social Determinants of Health Research Center, Lorestan University of Medical Sciences, Khorramabad, Iran ease is diagnosed in children by whooping coughs and vomiting. In adults, the symptoms are atypical in the form

$\uparrow$ What is "already known" in this topic:

The number of cases with pertussis has been reduced considerably. It is indicated a remarkable success of Iran in preventing pertussis.

$\rightarrow$ What this article adds:

Some of the provinces in the country will be facing with higher risk of developing the disease by 2021. Accordingly, appropriate planning for prevention, interventional programs, and also a more accurate implementation of the vaccination programs against the disease in these provinces are necessary. 
of long coughs. It is one of the major causes of child mortality worldwide (1). The disease declined by more than $90 \%$ following the implementation of widespread vaccination programs in the 1950s and 1960s. However, between 20 and 40 million cases of pertussis are annually reported worldwide, with $90 \%$ being in the developing countries $(1,2)$.

Therefore, despite widespread vaccination and underreporting, there is an increase in the incidence of the disease in neonates, children, and adults worldwide. Also, despite a relatively high vaccination coverage, the disease has been one of the emerging diseases in recent years (3). In the United States in 2012, the diagnosed cases of B. Pertussis was more than that in 1955 (4). In the UK in 2013, there was a significant increase in the incidence of the disease compared to the 1970s and 1980s. The 2 hypotheses raised for the increase in the incidence of the disease in recent years could be low vaccination coverage and susceptibility to infection despite vaccination (5). In a study performed in Iran in 2015, most confirmed cases of pertussis were in Tehran (59 cases), Mazandaran (45 cases), East Azerbaijan (28 cases), and Ilam (18 cases) provinces (6).

Studies conducted in Iran and around the world have examined pertussis from different angles. The evidence shows that, in terms of the efficacy and immunogenicity of pertussis vaccine, passive and normal immunity and sources of infection for neonates as well as severity of injury to this group indicate that the incidence of pertussis needs special attention.

Based on the evidence cited, it can be stated that pertussis is an endemic disease in Iran or perhaps even in most countries. The reported cases of pertussis based on the status and nature of the disease care system across the Iranian provinces in different years show significant fluctuations and variations that can make it difficult to understand the annual incidence trend or even incidence in different regions of Iran. Therefore, it should be examined continuously $(7,8)$.

As the incidence of the disease varies in different Iranian provinces, understanding the geographical pattern of its incidence and prevalence can be very important for interventions and disease management. Disease mapping using the geographic information system (GIS) has been performed around the world for many years. It is now considered as a first step in the development of disease warning systems and its importance is increasing day by day (8$10)$.

It is important to use the GIS to manage health systems and infrastructure. The GIS is a powerful set of tools for storing and retrieving data and converting and displaying spatial data from the real world (11). Therefore, the purpose of this study was to investigate the incidence and geographical distribution of pertussis using the GIS and to predict its incidence in Iran in 2021.

\section{Methods}

This was a descriptive analytical study with applied results. The research uses spatial and climatic information and incidence rate of typhoid in Iran. Initially, the infor- mation on pertussis was obtained from the Center for Communicable Diseases Control during 2005-2015. The incidence and frequency table for pertussis was prepared for each year and the incidence rate was calculated for 100,000 people in Iran. In the next step, the GIS was used to prepare geographic maps of the disease incidence and frequency. To prepare the spatial database for the disease, the vector map of Iran's administrative divisions for 2017 used by the National Mapping Organization was used to link the descriptive information about the disease to the spatial data in the GIS environment. The geographical locations were verified through Google Earth. When the geographic database of the disease was developed, descriptive information, such as incidence and frequency of the disease, was added to the GIS descriptive table using Excel. Next, a spatial analysis was prepared to map the incidence and frequency distribution of disease during 2005-2015 in the GIS environment through symbology functions. With the information of each province, the incidence and frequency map of the disease was prepared for the whole country. To plot the disease prediction map, it was assumed that the probability of disease occurrence was higher in regions with the highest incidence and the highest recurrence of disease in a statistical period. Therefore, using the Raster Calculator tool, the fuzzy map of the disease over a given period was multiplied by the disease recurrence map and the disease prediction map was eventually drawn (12). The map shows the most likely areas for disease incidence in red.

With the information of each province available, the incidence and frequency of the disease was developed for the whole country. To plot the map of interpolation, the inverse distance interpolation method was used (13).

\section{High-risk Points Analysis}

The Getis-Ord-Gi* statistic was used for appropriate spatial distribution of hot and cold spots. A disease is recognized as a hot spot when its figures and those of its surrounding conditions are fairly high. When the Getis-OrdGi* statistic is calculated to be 1,2 , and 3 , the CI is estimated at $99 \%, 95 \%, 90 \%$, respectively (14).

As the incidence data are obtained based on the findings of routine healthcare system, the difference in incidence that was observed in different provinces can be largely dependent on the sensitivity of the healthcare system to record and report cases in these provinces.

\section{Results}

The results showed that $51.6 \%$ of pertussis patients were men, and $70.8 \%$ were urban dwellers. It also showed that $77 \%$ of the cases recovere d without any complication (Table 1).

The results of the study showed that most pertussis cases reported between 2008 and 2015 were in 2013 with 1443 cases (Fig. 1).

The results show that most pertussis cases during 20092015 were reported in April and June (Fig. 2).

The results showed that the highest incidence of pertussis during 2009-2015 was in Zanjan, Qom, Mazandaran, and Qazvin provinces (Table 2). 


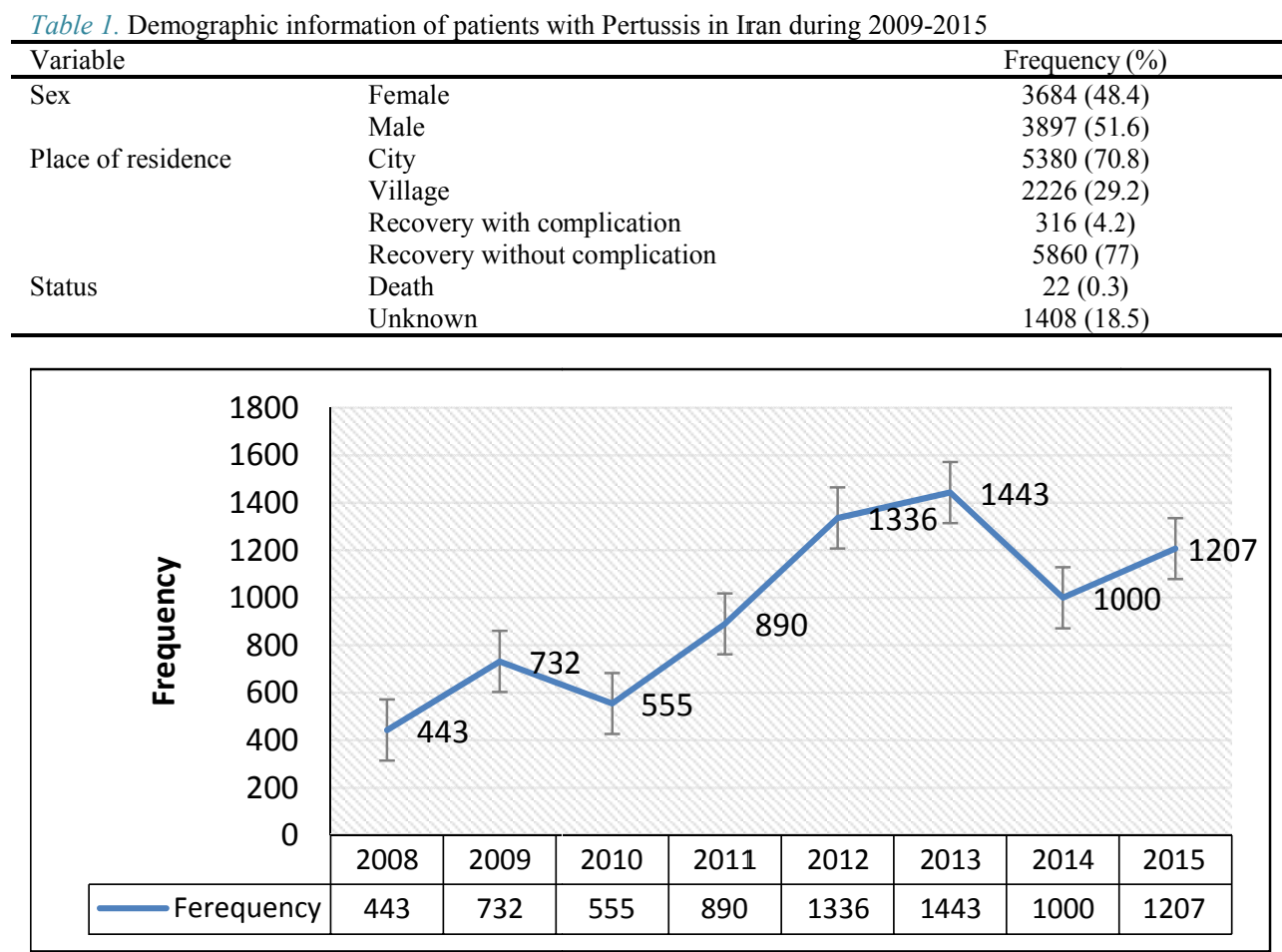

Fig. 1. Frequency of reported cases of pertussis during 2008-2015

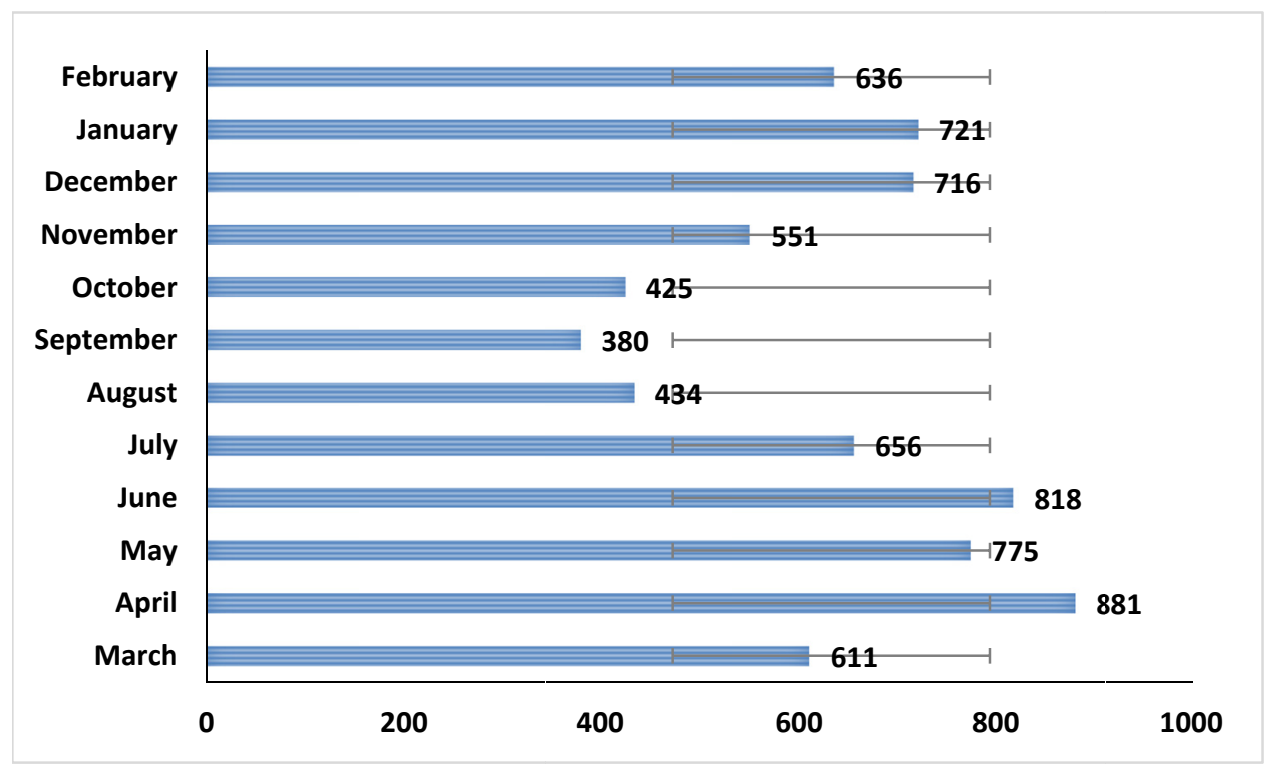

Fig. 2. Pertussis frequency according to the months reported

The results indicated that the incidence of pertussis in Iran increased from 0.74 in 2009 to 1.53 in 2015 (Fig. 3).

The prepared GIS maps show the incidence of pertussis in different provinces during 2009-2015 (Fig. 4).

According to the latest information on the incidence of the disease (incidence in 2015), Mazandaran, Qazvin, Qom, and Zanjan provinces had the highest incidence in 2015.

Based on the GIS maps of the whole country, $5.04 \%$ of the total area of Iran $\left(83022.79 \mathrm{~km}^{2}\right)$ comprising parts of Isfahan, Alborz, Ilam, East Azarbaijan, Tehran, Khorasan

Razavi, Zanjan, Semnan, Fars, Qazvin, Qom, Kurdistan, Kermanshah, Golestan, Gilan, Mazandaran, Markazi, Hormozgan, Hamedan, and Y'azd provinces are at high risk for pertussis in the coming years (2021). Based on the modeling results in Iran, Qom, Mazandaran, Tehran, Qazvin, and Zanjan provinces with, 76.76\%, 73.69\%, $66.32 \%, 30.94 \%$ and $24.18 \%$ of their areas $\left(\mathrm{Km}^{2}\right)$, respectively, are at high risk for pertussis in the coming years (Fig. 5). 


\begin{tabular}{|c|c|c|c|c|c|c|c|c|}
\hline Row & Province & 2009 & 2010 & 2011 & 2012 & 2013 & 2014 & 2015 \\
\hline 1 & East Azerbaijan & 0.24 & 0.51 & 1.13 & 2.00 & 3.94 & 2.84 & 3.23 \\
\hline 2 & West Azerbaijan & 0.00 & 0.33 & 0.10 & 0.51 & 0.35 & 0.28 & 0.22 \\
\hline 3 & Ardebil & 0.00 & 0.16 & 0.24 & 0.64 & 0.63 & 0.63 & 0.23 \\
\hline 4 & Isfahan & 0.04 & 0.46 & 0.49 & 0.98 & 0.87 & 0.78 & 1.72 \\
\hline 5 & Alborz & 0.00 & 0.26 & 1.08 & 1.51 & 1.48 & 1.45 & 1.66 \\
\hline 6 & Ilam & 0.00 & 1.08 & 1.08 & 3.20 & 2.11 & 1.39 & 1.55 \\
\hline 7 & Bushehr & 0.41 & 0.60 & 0.29 & 0.47 & 0.74 & 1.27 & 1.78 \\
\hline 8 & Tehran & 0.73 & 0.91 & 1.37 & 1.84 & 2.30 & 1.11 & 1.91 \\
\hline 9 & Chaharmahal va Bakhtiari & 1.48 & 1.47 & 1.68 & 0.77 & 1.64 & 1.84 & 3.86 \\
\hline 10 & Southern Khorasan & 2.30 & 3.04 & 0.90 & 3.78 & 1.87 & 3.16 & 1.17 \\
\hline 11 & Khorasan Razavi & 0.53 & 0.24 & 0.72 & 0.53 & 0.73 & 0.67 & 1.02 \\
\hline 12 & Northern Khorasan & 0.12 & 0.23 & 1.50 & 2.16 & 1.13 & 0.22 & 0.88 \\
\hline 13 & Khuzestan & 0.79 & 0.78 & 0.73 & 0.74 & 1.80 & 1.12 & 1.29 \\
\hline 14 & Zanjan & 0.30 & 0.30 & 3.94 & 7.02 & 8.29 & 4.11 & 4.82 \\
\hline 15 & Semnan & 2.61 & 0.80 & 0.95 & 0.94 & 2.61 & 0.76 & 1.49 \\
\hline 16 & Sistan and Baluchestan & 0.36 & 0.36 & 0.63 & 0.15 & 0.11 & 0.22 & 0.11 \\
\hline 17 & Fars & 0.40 & 0.18 & 0.33 & 0.19 & 0.28 & 0.21 & 0.08 \\
\hline 18 & Qazvin & 2.04 & 1.43 & 5.41 & 3.79 & 4.57 & 4.52 & 5.12 \\
\hline 19 & Qom & 7.04 & 0.80 & 4.43 & 6.66 & 4.19 & 4.86 & 4.29 \\
\hline 20 & Kurdistan & 0.75 & 0.34 & 1.34 & 4.06 & 2.84 & 2.30 & 2.80 \\
\hline 21 & Kerman & 0.07 & 0.07 & 0.20 & 0.17 & 0.59 & 0.81 & 0.32 \\
\hline 22 & Kermanshah & 1.41 & 0.52 & 1.54 & 1.69 & 4.03 & 1.32 & 0.76 \\
\hline 23 & Kohgiloyeh and Boyerahmad & 0.31 & 0.15 & 0.30 & 1.49 & 0.59 & 0.14 & 0.00 \\
\hline 24 & Golestan & 0.29 & 0.69 & 0.62 & 1.38 & 1.79 & 0.27 & 0.84 \\
\hline 25 & Gilan & 0.20 & 0.16 & 0.44 & 0.40 & 0.36 & 0.55 & 0.24 \\
\hline 26 & Lorestan & 0.06 & 0.57 & 0.97 & 0.68 & 0.73 & 0.56 & 0.11 \\
\hline 27 & Mazandaran & 2.66 & 4.53 & 5.34 & 10.35 & 7.42 & 4.94 & 5.41 \\
\hline 28 & Markazi & 1.51 & 0.93 & 0.35 & 0.56 & 0.69 & 0.27 & 0.27 \\
\hline 29 & Hormozgan & 0.13 & 0.91 & 0.06 & 0.31 & 0.18 & 0.48 & 0.23 \\
\hline 30 & Hamedan & 1.56 & 1.09 & 1.88 & 2.09 & 1.80 & 1.06 & 1.56 \\
\hline \multirow[t]{2}{*}{31} & Yazd & 1.44 & 0.66 & 1.12 & 3.12 & 1.91 & 1.59 & 0.64 \\
\hline & Whole country & 0.74 & 0.74 & 1.18 & 1.75 & 1.88 & 1.28 & 1.53 \\
\hline
\end{tabular}

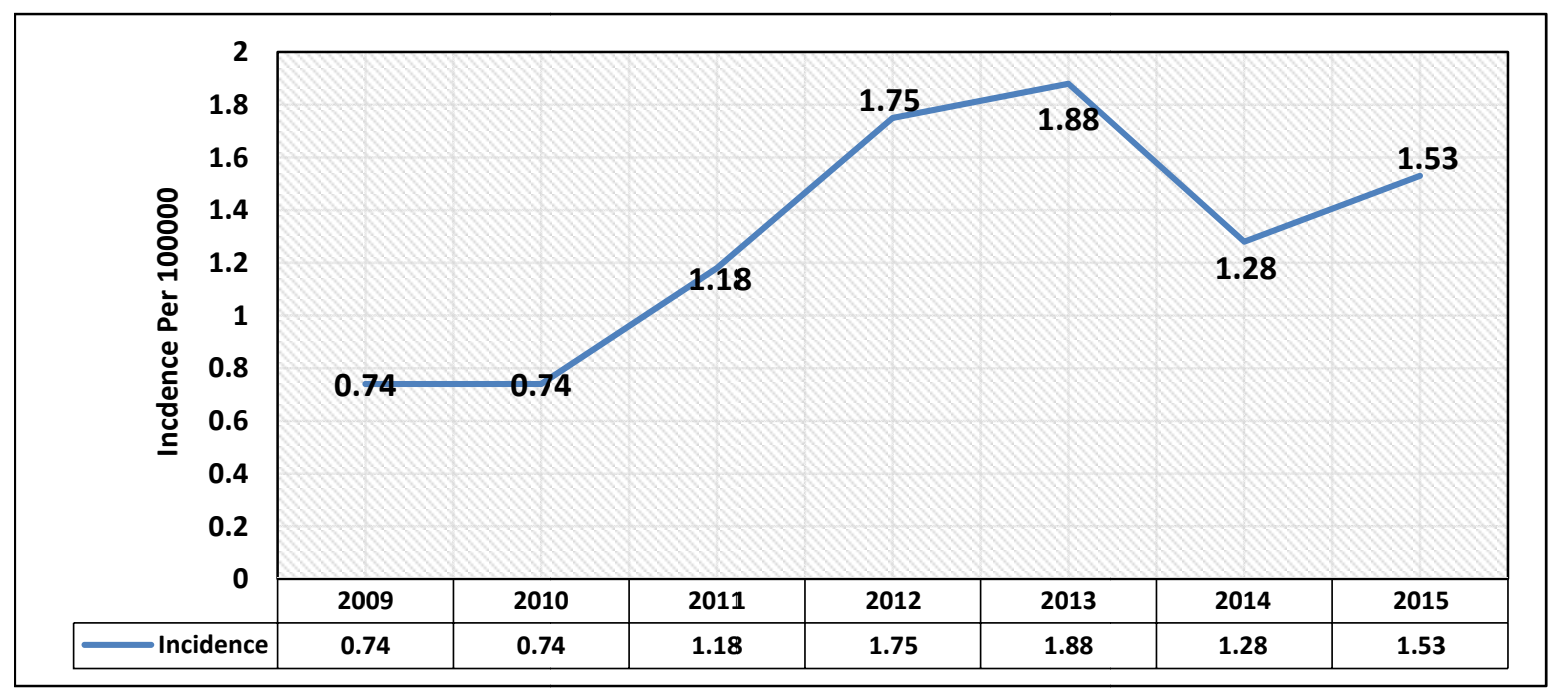

Fig. 3. Trend of pertussis incidence in Iran during 2009-2015

\section{Discussion}

Despite high vaccination coverage in most countries, the increase in cases of pertussis in infants, adolescents, and adults in recent years has attracted many countries' attention. As a result, it is considered as a recurring disease that threatens public health $(15,16)$.

The results of this study showed that the highest incidence of pertussis during 2009-2015 was in Zanjan, Qom, Mazandaran, and Qazvin provinces. The incidence of pertussis in Iran increased from 0.74 in 2009 to 1.53 in 2015. Based on the modeling results for Iran, Qom, Mazandaran, Tehran, Qazvin, and Zanjan provinces, with $76.76 \%$,
$73.69 \%, 66.32 \%, 30.94 \%$ and $24.18 \%$ of their areas, are at high risk for pertussis in the coming years, respectively.

The results of our study showed that Zanjan, Qom, Mazandaran, and Qazvin provinces had the highest incidence of the disease during 2009-2015. A cross-sectional study in Zanjan investigated 123 people with coughing symptoms for more than 2 weeks without any other disease, from which 23 cases $(18.7 \%)$ were diagnosed with pertussis (17).

In a study by Ghorbani et al, out of 9613 suspected cases of pertussis reported from Iranian provinces, laboratory results were positive in $6.6 \%$ of cases. Most suspected 


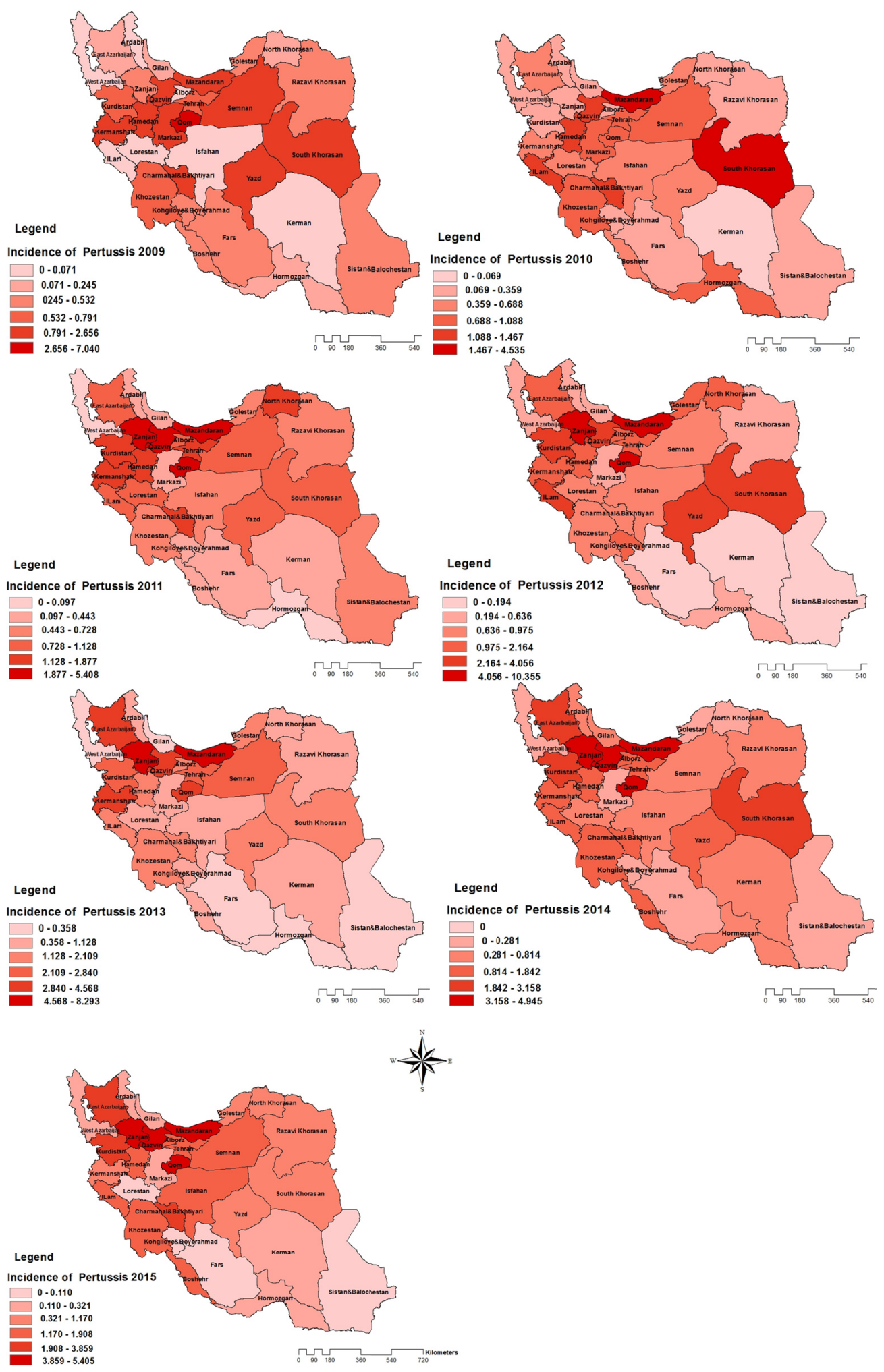

Fig. 4. Pertussis incidence in Iran during 2009-2015

cases of pertussis were reported from Mazandaran and Tehran provinces, but the ratio of confirmed cases was higher in Alborz and South Khorasan than in other Iranian provinces (18). In the study of Bellettini et al, 222 suspected pertussis patients were studied from September
2011 to January $2013,72.5 \%$ of the cases were positive and $60.9 \%$ were under the age of 1 (19). In a study in Mexico, of 147 suspected patients, 59 (40.1\%) were positive(18). In a South Korean study of 490 patients, 34 (6.9\%) cases were positive (19). 


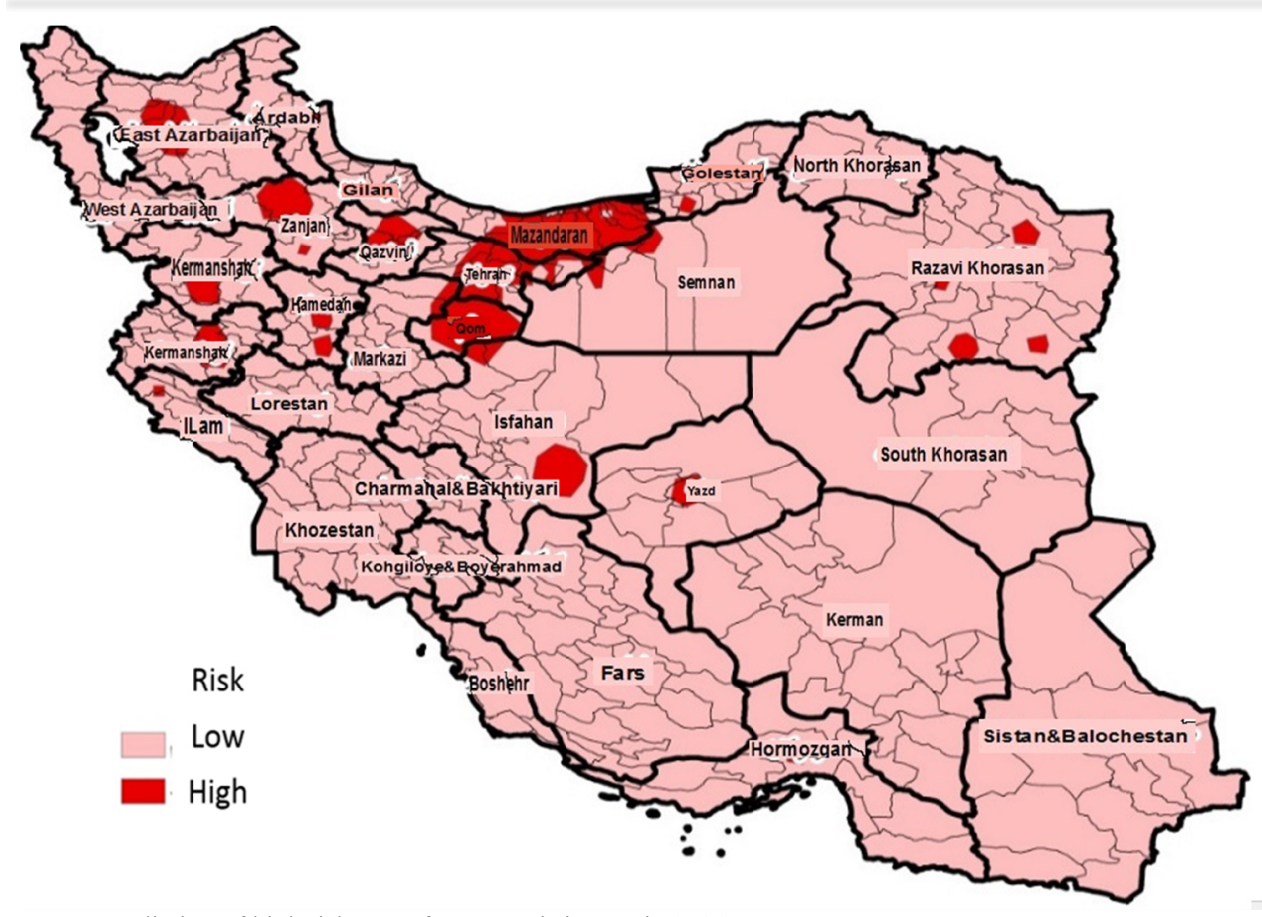

Fig. 5. Prediction of high risk areas for pertussis in Iran in 2021

The reasons for these differences may be in the sensitivity and specificity of the pertussis disease care systems, the definitions used in the care systems for suspected and probable cases, sampling and its transportation, the resources available for testing, the status of immunization coverage, and the endemic conditions of the disease in different regions. In recent decades, there has been an increase in the prevalence of the disease, which indicates that pertussis is one of the recurrent diseases worldwide. In the United States, the prevalence of the disease was $63.4 \%$ in the $1980 \mathrm{~s}, 88.7 \%$ in the $1990 \mathrm{~s}$, and the highest incidence was during 2001-2003 (20).

The incidence of pertussis in Iran increased from 0.74 in 2009 to 1.53 in 2015 . There are several reasons for this increased trend, including decreased immunity after vaccination over time, failure in complete vaccination, source of infection for infants, source of infection for adults, presence of polymorphism strains due to gene mutations in vaccine-associated antigens or ineffective vaccines, and improvements in disease diagnosis and infection reporting. In recent years in Iran, the collaboration between the Center for Disease Management and the Pasteur Institute of Iran, as a national laboratory for pertussis, the laboratory diagnosis of the disease has made significant progress, which may justify the increased incidence of disease dbecause of the progress in diagnosis.

Based on the statistics of the Ministry of Health's Center for Communicable Diseases Management, nowadays, the vaccination coverage in Iran is $99 \%$. However, our modeling results showed that Qom, Mazandaran, Tehran, Qazvin, and Zanjan provinces with $76.76 \%, 73.69 \%$, $66.32 \%, 30.94 \%$, and $24.18 \%$ of their areas, respectively, are at high risk for pertussis in the coming years.

The factors that may be a risk for these provinces include incomplete vaccination, genetic variation of strains, possibility of the difference in recent pathogenic strains, and adult carriers transmitting the disease. Therefore, proper implementation of the vaccination program, identification of pathogenic strains and treatment of carrier individuals in these provinces can prevent the incidence of the disease in the coming years.

\section{Conclusion}

Based on the results, the incidence of the disease has been increasing in recent years, indicating the emergence of the disease in Iran. The modeling maps show that the Iranian provinces of Qom, Tehran, Zanjan, and Qazvin are at risk of the disease incidence in the coming years, indicating the need for planning and more precise implementation of the vaccination programs against the disease. Proper interventions can help prevent the disease from occurring in the years to come and minimize its incidence.

\section{Acknowledgment}

This paper is based on the results of a research project with the ethical of code IR.MUK.REC.1395.184 approved by Kurdistan University of Medical Sciences. The close cooperation of the Center for Communicable Diseases Management, Ministry of Health and Medical Education, distinguished deputies of health affairs of the medical sciences universities, responsible experts and specialists in the prevention and fighting of the infectious diseases groups of Iranian provinces, experts, technicians, specialists, and the staff at the health centers and health houses of Iran, as well as the collaboration of all those involved in the project are greatly appreciated.

\section{Conflict of Interests}

The authors declare that they have no competing interests. 


\section{References}

1. de Greeff SC, Dekkers AL, Teunis P, Rahamat-Langendoen JC, Mooi FR, de Melker HE. Seasonal patterns in time series of pertussis. Epidemiol Infect. 2009;137(10):1388-95.

2. Mooi FR, Van Loo I, King AJ. Adaptation of Bordetella pertussis to vaccination: a cause for its reemergence? Emerg Infect Dis. 2001;7(3 Suppl):526.

3. Rumbo M, Hozbor D. Development of improved pertussis vaccine. Hum Vaccines Immunother. 2014;10(8):2450-3.

4. Jackson DW, Rohani P. Perplexities of pertussis: recent global epidemiological trends and their potential causes. Epidemiol Infect. 2014;142(4):672-84.

5. Althouse BM, Scarpino SV. Asymptomatic transmission and the resurgence of Bordetella pertussis. BMC Med. 2015;13(1):146.

6. Ghorbani G, Zahraei S, Doosti F, Moosazadeh M. Epidemiological pattern of bordetella pertussis in Iran, 2011-2013. J Mil Med. 2016;17(4):215-22.

7. Bellettini CV, de Oliveira AW, Tusset C, Baethgen LF, Amantéa SL, Motta F, et al. Clinical, laboratorial and radiographic predictors of Bordetella pertussis infection. Rev Paul Pediatr. 2014;32(4):292-8.

8. Tanser F, Bärnighausen T, Cooke GS, Newell M-L. Localized spatial clustering of HIV infections in a widely disseminated rural South African epidemic. Int J Epidemiol. 2009;38(4):1008-16.

9. Zhi-Hang P, Yue-Jia C, Reilly KH, Lu W, Qian-Qian Q, Zheng-Wei $\mathrm{D}$, et al. Spatial distribution of HIV/AIDS in Yunnan province, People's Republic of China. Geospat Health. 2011;5(2):177-82.

10. Pordanjani SR, Atamaleki A, Amiri M, Khazaei Z, Fallahzadeh H, Alayi R, et al. Study on epidemiological status, spatial and temporal distribution of human brucellosis in kohgiluyeh and Boyer-Ahmad Province during 2011-2017. Adv Hum Biol. 2020;10(1):22.

11. Seif A, Rashidi M, Rozbahani R, Daheshti N, Poursafa P. GIS application in medical researches a solution for prevention from disease. J Esfahan Univ Med Sci. 2011;29(164):1-10.

12. Khoshdel A, Nouri Fard M, Pezeshkn R, AR. SM. Mapping of important diseases communicable in Iran. Health Develop J. 2012;1(1):31-46.

13. Setianto A, Setianto A, Triandini T, Triandini T. Comparison of kriging and inverse distance weighted (IDW) interpolation methods in lineament extraction and analysis. J Appl Geol. 2013;5(1):21-9.

14. Getis A, Ord JK. The analysis of Spatial Association by use of Distance Statistics. Geogr Anal. 1992;24(3):189-206.

15. Heininger U, Cherry JD. Pertussis immunisation in adolescents and adults-Bordetella pertussis epidemiology should guide vaccination recommendations. Expert Opin Biol Ther. 2006;6(7):685-97.

16. Melvin JA, Scheller EV, Miller JF, Cotter PA. Bordetella pertussis pathogenesis: current and future challenges. Nat Rev Microbiol. 2014;12(4):274-88.

17. Jozpanahi M, Mobaien A, Karami A, Hamzelo S. Frequency of pertussis among adults with prolonged cough. Jundishapur Sci Med J. 2011;10(4):395-401.

18. Ochoa-Perez UR, Hernández-Sierra JF, Escalante-Padrón FJ, Contreras-Vidales S, Berman-Puente AM, Hernandez-Maldonado F, et al. Epidemiology of Bordetella pertussis in San Luis Potosi, Mexico. Pediatr Infect Dis J. 2014;33(5):540-2.

19. Park S, Lee SH, Seo KH, Shin KC, Park YB, Lee MG, et al. Epidemiological aspects of pertussis among adults and adolescents in a Korean outpatient setting: a multicenter, PCR-based study. J Korean Med Sci. 2014;29(9):1232-9.

20. Wood N, McIntyre P. Pertussis: review of epidemiology, diagnosis, management and prevention. Paediatr Respir Rev. 2008;9(3):201-12. 